\title{
Correction to: Ghrelin Fights against Titanium Particle-Induced Inflammatory Osteolysis through Activation of $\beta$-Catenin Signaling Pathway
}

Ruize Qu, ${ }^{1,2}$ Xiaomin Chen, ${ }^{1,2}$ Yongjian Yuan, ${ }^{3}$ Wenhan Wang, ${ }^{2}$ Cheng Qiu, ${ }^{2}$ Long Liu, ${ }^{1}$ Peng Li, ${ }^{1}$ Zhaoyang Zhang, ${ }^{4}$ Krasimir Vasilev, ${ }^{5}$ Liang Liu, ${ }^{5}$ John Hayball, ${ }^{6,7}$ Yunpeng Zhao, ${ }^{3,8}$ Yuhua $\mathrm{Li}^{3,8}$ and Weiwei $\mathrm{Li}^{1,8}$

Correction to: Inflammation (2019) 42(5):1652-1665

https://doi.org/10.1007/s10753-019-01026-w

The original version of this article was published with incorrect Fig. 1B. The correct Fig. 1B is now presented in Fig. 1 shown at the next page.

Ruize Qu, Xiaomin Chen and Yongjian Yuan contributed equally to this work.

The online version of the original article can be found at https://doi.org/ 10.1007/s10753-019-01026-w

\footnotetext{
${ }^{1}$ Department of Pathology, Qilu Hospital, Shandong University, Jinan, China

${ }^{2}$ Cheeloo College of Medicine, Shandong University, Jinan, China

${ }^{3}$ Department of Orthopedics, Qilu Hospital, Shandong University, 107 Wenhuaxi Rd., Jinan, 250012, Shandong, People's Republic of China

${ }^{4}$ Department of Emergency Surgery, Qilu Hospital, Shandong University, Jinan, 250012, Shandong, People's Republic of China

${ }^{5}$ School of Engineering, University of South Australia, Mawson Lakes, SA 5095, Australia

${ }^{6}$ Experimental Therapeutics Laboratory, Hanson Institute, and Sansom Institute for Health Research, School of Pharmacy and Medical Science, University of South Australia, Adelaide, SA, Australia

${ }^{7}$ Robinson Research Institute, Adelaide Medical School, The University of Adelaide, Adelaide, SA, Australia
} 
a

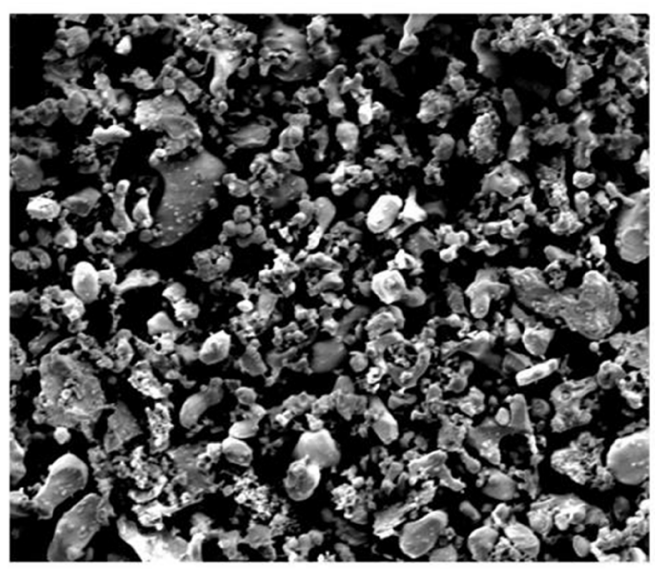

b

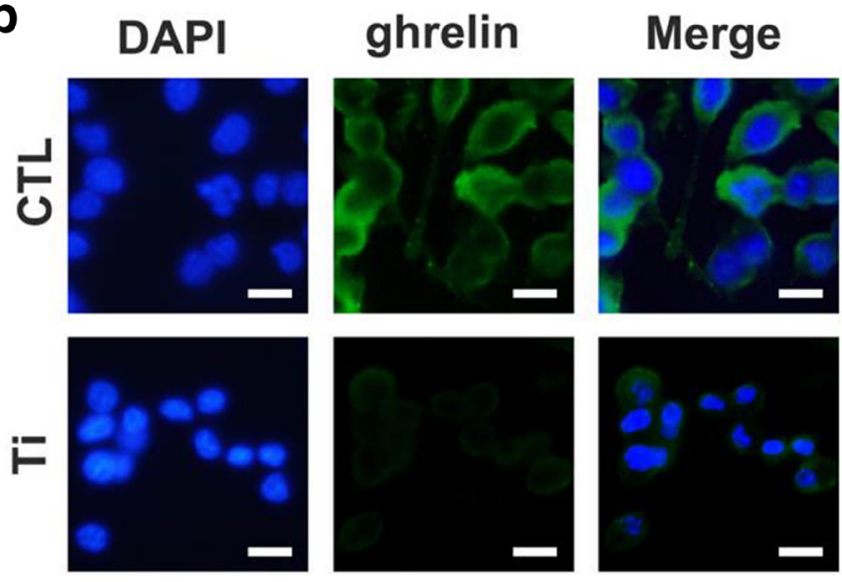

C

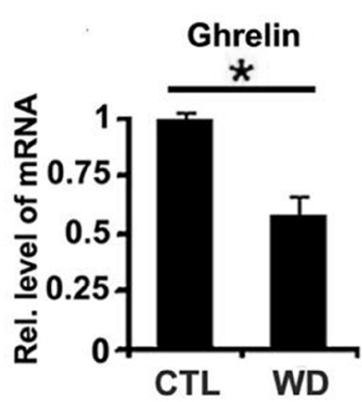

e

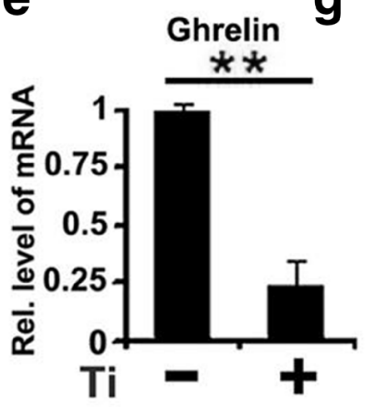

d

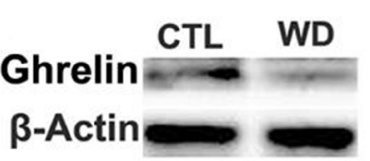

f

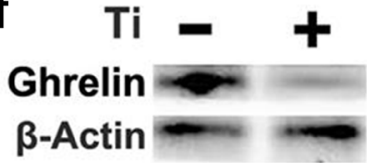

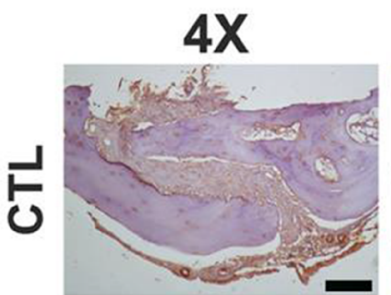
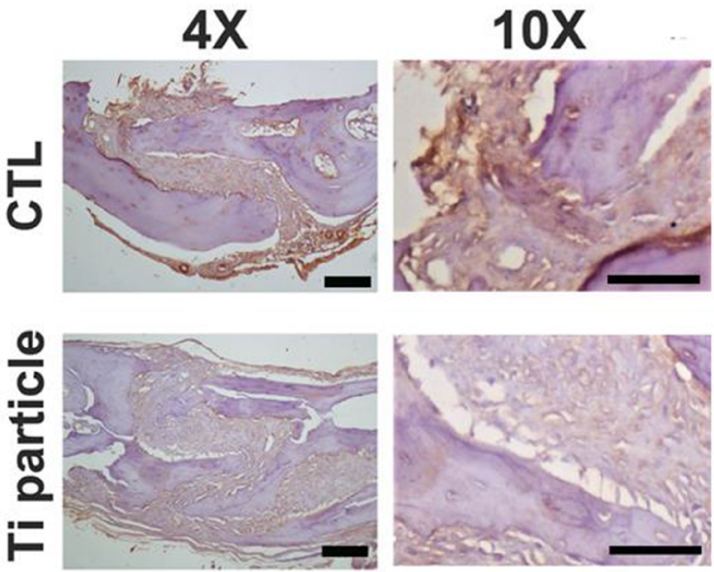

Fig. 1. Stimulation of Ti particles diminished ghrelin expression in vivo and ex vivo. a Scanning election microscopy (SEM) appearance of titanium particles (magnification $\times 5000$ ). b Stimulation of Ti apparently decreased ghrelin expression in MC3T3 cells, as measured by immunofluorescence. $\mathbf{c} \mathrm{mRNA}$ was extracted from mouse models, and real-time PCR was conducted. Expression of ghrelin decreased with the stimulation of wear debris. $\mathbf{d}$ Total protein was obtained from mouse models, and western blot showed that ghrelin expression was inhibited with the stimulation of wear debris. e Expression of ghrelin diminished with the stimulation of Ti particles, as indicated by real-time PCR. f Ghrelin expression was downregulated with Ti particle stimulation, as assessed via western blot. $\mathbf{g}$ Ghrelin secretion was diminished with the stimulation of Ti particles in vivo, as indicated by immunohistochemistry. $\left({ }^{*} p<0.05\right.$, $* * p<0.01 v s$ the control group). Scale bar: $150 \mu \mathrm{m}$. 